\title{
Toxic Shock Syndrome
}

National Cancer Institute

\section{Source}

National Cancer Institute. Toxic Shock Syndrome. NCI Thesaurus. Code C35498.

A rare acute life-threatening systemic bacterial noncontagious illness caused by any of several related staphylococcal exotoxins. It is characterized by high fever, hypotension, rash, multi-organ dysfunction, and cutaneous desquamation during the early convalescent period. The toxins affect the host immune system, causing an exuberant and pathological host inflammatory response. Laboratory findings include leukocytosis, elevated prothrombin time, hypoalbuminemia, hypocalcemia, and pyuria. 